\title{
Multi-laboratory efforts for the standardization of performance assessment of diffuse optics instruments - The BitMap Exercise
}

Pranav Lanka ${ }^{1}$, Lin Yang ${ }^{3}$, David Orive-Miguel ${ }^{4}$, Joshua Deepak Veesa ${ }^{5}$, Susanna Tagliabue ${ }^{6}$, Aleh Sudakou $^{7}$, Saeed Samaei ${ }^{7}$, Mario Forcione ${ }^{8}$, Zuzana Kovacsova ${ }^{9}$, Anurag Behera ${ }^{1}$, Thomas Gladytz ${ }^{3}$, Dirk Grosenick $^{3}$, Lionel Hervé ${ }^{4}$, Giuseppe LoPresti ${ }^{6}$, Lorenzo Cortese ${ }^{6}$, Turgut Durduran ${ }^{6}, K_{\text {Karolina Bejm }}^{7}$, Magdalena Morawiec ${ }^{7}$, Michał Kacprzak ${ }^{7}$, Piotr Sawosz ${ }^{7}$, Anna Grega ${ }^{7}$, Adam Liebert ${ }^{7}$, Antonio Belli ${ }^{8}$, Ilias Tachtsidis ${ }^{9}$, Frederic Lange ${ }^{9}$, Gemma Bale ${ }^{9}$, Luca Baratelli ${ }^{10}$, Sylvain Gioux ${ }^{10}$, Kalyanov Alexander ${ }^{11}$,

Martin Wolf ${ }^{11}$, , Sanathana Konugolu Venkata Sekar ${ }^{12}$, Marta Zanoletti ${ }^{1}$, Ileana Pirovano ${ }^{1}$, Michele Lacerenza $^{1}$, Lina Qiu ${ }^{1}$, Edoardo Ferocino ${ }^{1}$, Giulia Maffeis ${ }^{1}$, Caterina Amendola ${ }^{1}$, Lorenzo Colombo ${ }^{1}$, Laura Di Sieno ${ }^{1}$, Rebecca Re ${ }^{1}$, Andrea Farina ${ }^{2}$, Lorenzo Spinelli ${ }^{2}$, Alberto Dalla Mora ${ }^{1}$, Davide Contini ${ }^{1}$, Paola Taroni $^{1}$, Alessandro Torricelli ${ }^{1}$, Hamid Dehghani ${ }^{5}$, Heidrun Wabnitz ${ }^{3}$, and Antonio Pifferi ${ }^{1}$

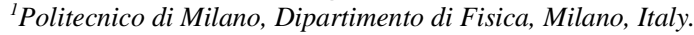

${ }^{2}$ Istituto di Fotonica e Nanotecnologie, Milano, Italy

${ }^{3} P T B$ - Physikalisch-Technische Bundesanstalt, Berlin, Germany. ${ }^{4}$ CEA, LETI, MINATEC Campus, France.

${ }^{5}$ School of Computer Science, University of Birmingham, Birmingham, UK.

${ }^{6}$ ICFO - The Institute of Photonic Sciences, Castelldefels, Spain.

${ }^{7}$ Nalecz Institute of Biocybernetics and Biomedical Engineering, Warsaw, Poland.

${ }^{8}$ National Institute for Health Research Surgical Reconstruction and Microbiology Research Centre, University Hospitals Birmingham, UK.

${ }^{9}$ Medical Physics \& Biomedical Engineering, UCL, London, UK

${ }^{10}$ University of Strasbourg, Strasbourg, France

${ }^{I I}$ Biomedical Optics Research Laboratory, Department of Neonatology, University Hospital Zurich, Zurich, Switzerland

${ }^{12}$ IPIC, Tyndall National Institute, Cork, Ireland;

sriramapranav.lanka@polimi.it

\begin{abstract}
Results from a multi laboratory initiative aiming for performance assessment of diffuse optics instruments are presented. A total of 29 diffuse optical instruments with varied applications from 11 partner institutions were enrolled in the study. 3 internationally accepted, standardized protocols were used to assess the performance of these instruments. The overarching methodology and future actions of the exercise will be briefly discussed.
\end{abstract}

\section{Methodology}

A wide range of diffuse optics instruments belonging mainly to the partners of a European level Marie Curie Consortium BITMAP ${ }^{1}$, covering different techniques (Continuous Wave, frequency domain, time domain) and applications (mammography, oximetry, functional imaging, tissue spectroscopy) were chosen for this performance assessment exercise. As a first step performance assessment tests were performed on the instruments based on 3 well accepted protocols which were followed by an initial comparison of the results. Future actions aim at deploying these measurements onto an open data repository and investigate common analysis tools for the whole dataset.

\section{Implementation}

\subsection{Instrumentation}

29 instruments at different TRL (technology readiness levels) were enrolled. Table 1 gives a brief summary of the different instruments enrolled, based on their applications and modalities.

Table 1. Instruments enrolled for the exercise

\begin{tabular}{cccccc}
\hline Application & \multicolumn{4}{c}{ Modality } & Total \\
\cline { 2 - 5 } & CW & TD & FD & MI & \\
\hline Spectroscopy & 1 & 9 & 1 & 0 & 11 \\
Imaging & 0 & 4 & 0 & 1 & 5 \\
Oximetry & 2 & 8 & 1 & 0 & 11 \\
DCS & 0 & 2 & 0 & 0 & 2 \\
Total & 3 & 23 & 2 & 1 & $\mathbf{2 9}$ \\
\hline
\end{tabular}


DCS = Diffuse Correlation Spectroscopy, CW = Continuous Wave, $\mathrm{TD}=$ Time Domain, FD = Frequency Domain, $\mathrm{MI}=$ Modulated Imaging

\subsection{Protocols and Phantoms}

The performance assessment was based on the following 3 protocols.

\begin{tabular}{|l|l|l|l|l|}
\hline Protocol & Tests & Phantoms & Measurable & Characterizes \\
\hline MEDPHOT $^{2}$ & $\begin{array}{l}\text { Accuracy, Linearity, } \\
\text { Uncertainty, Stability, } \\
\text { Reproducibility }\end{array}$ & $\begin{array}{l}\text { Matrix of 32 } \\
\text { homogeneous } \\
\text { phantoms }\end{array}$ & $\begin{array}{l}\text { Absolute absorption }\left(\mu_{a}\right) \\
\text { and reduced scattering } \\
\left(\mu^{\prime}\right) \text { coefficients }\end{array}$ & $\begin{array}{l}\text { Ability to accurately } \\
\text { retrieve absolute optical } \\
\text { properties }\end{array}$ \\
\hline BIP $^{3}$ & $\begin{array}{l}\text { General performance, } \\
\text { Responsivity, DNL }\end{array}$ & Responsivity & $\begin{array}{l}\text { IRF, Background, DNL, } \\
\text { Responsivity }\end{array}$ & $\begin{array}{l}\text { General instrument } \\
\text { performance }\end{array}$ \\
\hline nEUROPt $^{4}$ & $\begin{array}{l}\text { Depth selectivity, lateral } \\
\text { resolution }\end{array}$ & $\begin{array}{l}\text { Solid } \\
\text { switchable }\end{array}$ & $\begin{array}{l}\text { Contrast, Contrast to } \\
\text { noise ratio }\end{array}$ & $\begin{array}{l}\text { Ability to detect an } \\
\text { inhomogeneity }\end{array}$ \\
\hline
\end{tabular}

\section{Results}

Fig 1. The absorption and reduced scattering coefficient spectra obtained by the different instruments on one of the 32 phantoms as a part of the first protocol: MEDPHOT. The legend corresponds to a unique identifier for each instrument enrolled. Not all instruments were eligible for all the tests.
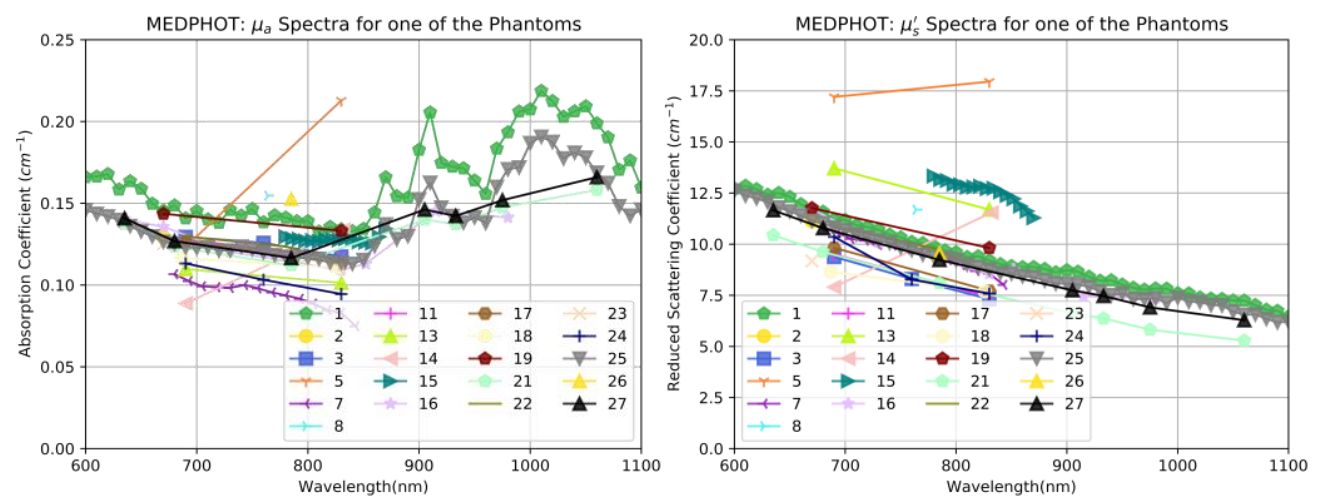

Figure 1 shows an example result of one of the performance assessment tests of the MEDPHOT protocol. Here we record the spectral response of the instruments when used to measure the optical properties of one of the 32 phantoms. The 3 protocols in total test multiple other aspects like the contrast detecting capabilities, linearity in the detector response, etc. On average, a total of 10 tests were performed on each instrument. Figure 1 also shows considerable variation in the retrieved optical properties which can partially be attributed to the diverse analysis methodologies employed to retrieve these results. The further actions of open data and use of common analysis tools mentioned earlier could be of great interest in overcoming these issues in the future. In the interest of space the results from the other tests have not been presented in this abstract.

\section{Conclusions:}

In Conclusion, 29 diffuse optics instruments from 11 different institutions across 7 EU nations were enrolled in a large-scale multi-laboratory performance assessment exercise. A total of 10 tests based on 3 international protocols were employed and the preliminary data analysis was performed. These results could give valuable insights into the advantages and shortcomings of the field of diffuse optics.

\section{References:}


[1] "BitMap ITN - Home," < http://www.bitmap-itn.eu/> (20 October 2019).

[2] Pifferi, A., et al., Applied Optics 44(11), 2104 (2005).

[3] Wabnitz, H., et al., Journal of Biomedical Optics 19(8), 086010 (2014).

[4] Wabnitz, et al., Journal of Biomedical Optics 19(8), 086012 (2014). 